\title{
Improving Dynamic Performance of Stewart Platforms through Optimal Design Based on Evolutionary Multi-objective Optimization Algorithms
}

\author{
Lv Bangjun \\ College of Power Engineering \\ Naval University of Engineering \\ Wuhan, China, 8627-83443264 \\ lvbangjun@gmail.com
}

\author{
Peng Likun \\ College of Power Engineering \\ Naval University of Engineering \\ Wuhan, China, 8627-83443264 \\ pelik@163.com
}

\author{
Ming Tingtao \\ Naval Equipment Repairing and \\ Supervising Room \\ Shanghai, China, 8621-58514781 \\ mtt1021@yahoo.com.cn
}

\begin{abstract}
The Stewart platform has characteristics of low natural frequency, antagonistic design objectives and always no analytical expression of both objective functions and constraints which make it difficult to obtain optimum performance with high dynamic performance. Compared with the traditional adopting indices such as manipulability, stiffness, dexterity, accuracy, etc., lowest natural frequency in the total workspace and ratio of maximum and minimum frequencies at typical configurations are developed as dynamic stability and isotropy indices respectively to improve the dynamic performance of Stewart platform. Evolutionary Multi-objective Optimization (EMO) was introduced synthetically considering the dynamic stability index, isotropy index and global dimensionally homogeneous Jacobian matrix condition number. Two typical EMO algorithms are applied for solving the Multi-objective Optimization Problem (MOP), and the algorithms performance was compared. The results are valuable in designing a Stewart platform for well dynamic capability.
\end{abstract}

Keywords-Stewart platform; dynamic performance; MOP; EMO; optimal design

\section{INTRODUCTION}

Parallel manipulators are widely accepted as ideal candidate in various applications for their inherent superior properties compared with their counterparts, such as higher rigidity, better positioning accuracy, high load capacity and simpler inverse kinematics. However, the relatively small workspace, complex input-output relationship and singularity problems in workspace advise caution in choosing a design [1].

It is well known that performances of parallel manipulators are heavily dependent upon the choice of the mechanical structure and even more from its dimensioning [2]. An appropriate design of geometric parameters in the early stage is of vital significance. Many scholars have paid close attention to the dimensioning optimum design of parallel manipulators [36]. On the one hand, different indices have been adopted for different applications. Gosselin has introduced the global conditioning index (GCI) as the average value of the inverse of the condition number over a given workspace to provide an overview of the performance of the robot over its workspace [7]. Nawratil proposed two new performance indices: an endeffector dependent index defined through operation ellipsoid and an end-effector independent index called control number (CTN). Optimal designs of 6-DoF and 3-DoF parallel manipulators are investigated based on the newly defined indices [8]. Jiang investigated the optimal design of generalized Gough-Stewart platforms based on an analytical dynamic isotropy measure proposed from the inverse mass matrix in joint space [9]. On the other hand, different methods have been applied. Lou investigated the effective regular workspace of parallel manipulators through controlled random search (CRS) technique which was proved efficient and reliable [1]. Wang proposed an optimal method to expand the bandwidth for the control of large hydraulic Stewart platform [10]. Hao proposed an interval analysis-based approach to solve optimal design problems and determined a design parameter space that satisfies all design requirements. Optimum is obtained by sampling the parameter space [11]. Recently, Gao optimized the stiffness and dexterity of Stewart manipulator based on artificial intelligence approaches [12]. Overall, it is apparent that many have concentrated on manipulability, stiffness, workspace volume, dexterity etc. Few optimization studies take natural frequencies into considerations which were proved as dynamic stability and isotropy index for Stewart platforms [9] [13].

\section{SYSTEM DESCRIPTION}

A parallel manipulator as shown in Fig. 1 is regarded as the Gough-Stewart manipulator which consists of a moving platform, a fixed base and six identical extensible links connecting the fixed base to moving platform. The kinematic chains associated with the six legs, from base to platform, consist of a universal joint (2-DOF), a cylinder, an actuated prismatic joint (1-DOF), a piston, and a spherical joint (3-DOF) attached to the moving platform. The platform has six DOF according to Kutzbach-Gruber formula. 


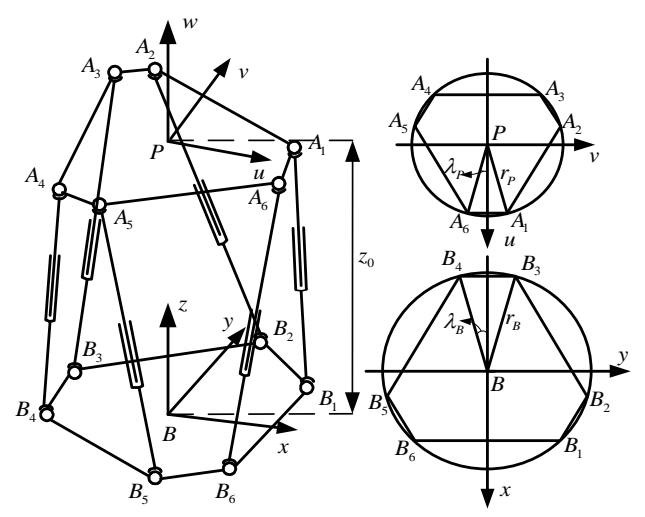

Figure.1 Schematic and design variables for Gough-Stewart manipulator

The architecture of such a manipulator can then be fully defined by design variables as shown in Fig. 1

$$
\boldsymbol{\alpha}=\left[R_{p}, R_{B}, \lambda_{p}, \lambda_{B}, z_{0}\right]
$$

where $R_{p}, R_{B}$ are the radius of the moving platform and the base, respectively, $\lambda_{p}, \lambda_{B}$ the half angle between two close attachment points on the platform and on the base, $z_{0}$ the height of platform at mid-stroke configuration. $\boldsymbol{\alpha} \in\left[\boldsymbol{\alpha}_{L}, \boldsymbol{\alpha}_{U}\right] . \boldsymbol{\alpha}_{L}, \boldsymbol{\alpha}_{U}$ are the lower and upper boundaries of these design variables considering the manufacture and machining factors.

\section{WORKSPACE CONSTRAINTS}

When design for parallel manipulators, we are aiming at a desired workspace $\boldsymbol{W}$ where the manipulator can work with no restriction. However, because of mechanical limits on passive joints, interference between links and limitations due to the actuators, not all configurations can be achieved. For Stewart manipulator, $\forall \boldsymbol{X} \in W, \boldsymbol{X}$ is an 6 dimensional vector denoted by $\boldsymbol{X}=[x, y, z, \varphi, \theta, \psi]$, where $x, y, z$ represent displacements of the moving reference frame centre $P$ and $\varphi, \theta, \psi$ represent Euler angles of the moving platform. The actuator lengths, deviation angles of passive joints (Fig. 2) and the distance between any pair of cylinders should satisfy

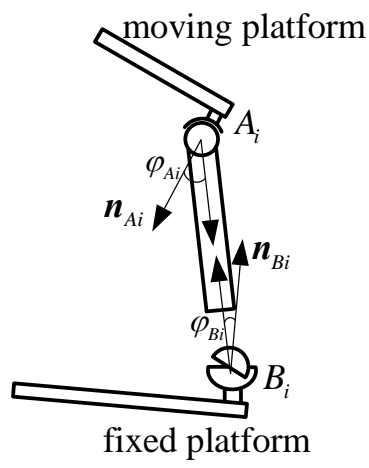

Figure.2 Deviation angles of passive joints

$$
\left\{\begin{array}{l}
L_{i}^{\min } \leq L_{i}(\boldsymbol{X}, \boldsymbol{\alpha}) \leq L_{i}^{\max } \\
\varphi_{A}^{\min } \leq \varphi_{A i}(\boldsymbol{X}, \alpha) \leq \varphi_{A}^{\max } \\
\varphi_{B}^{\min } \leq \varphi_{B j}(\boldsymbol{X}, \alpha) \leq \varphi_{B}^{\max } \\
\text { distance }\left(\boldsymbol{L}_{i}, \boldsymbol{L}_{j}\right) \geq R_{i}+R_{j}
\end{array} \quad i, j=1,2, \cdots 6\right.
$$

where $L_{i}^{\min }$ and $L_{i}^{\max }$ are the minimum and maximum lengths that can be reached by actuators respectively. $\varphi_{A(B)}^{\min }$ and $\varphi_{A(B)}^{\max }$ are the minimum and maximum deviation angles that can be reached by passive joints. Cylinders with radius of $R_{i(j)}$ denoted by line segments $\boldsymbol{L}_{i(j)}$.

\section{Global Dimensionally Homogeneous Jacobian MATRIX CONDITION NUMBER}

Salisbury and Craig introduced condition number (CDN) of Jacobian matrix to evaluate the dexterity of Stanford/JPL robot hand [14].However, when manipulators have both translational and rotational dofs, the condition number will not be invariant under scaling of dimensions. Use of the condition number of the Jacobian matrix with nonhomogeneous physic units in the optimal design or control may cause significant problems [15]. Kim and Ryu presented a new formulation of a dimensionally homogenous Jacbobian matrix for parallel manipulators with a planar mobile platform by using three end-effector points that are coplanar with the mobile platform joint. The dimensionally homogeneous Jacobian matrix has the form [16]

$$
\tilde{\boldsymbol{J}}=\left[\begin{array}{cccccc}
\frac{\partial l_{1}}{\partial A_{1 x}} & \frac{\partial l_{1}}{\partial A_{1 y}} & \frac{\partial l_{1}}{\partial A_{2 x}} & \frac{\partial l_{1}}{\partial A_{2 y}} & \frac{\partial l_{1}}{\partial A_{3 x}} & \frac{\partial l_{1}}{\partial A_{3 y}} \\
\frac{\partial l_{2}}{\partial A_{1 x}} & \frac{\partial l_{2}}{\partial A_{1 y}} & \frac{\partial l_{2}}{\partial A_{2 x}} & \frac{\partial l_{2}}{\partial A_{2 y}} & \frac{\partial l_{2}}{\partial A_{3 x}} & \frac{\partial l_{2}}{\partial A_{3 y}} \\
\frac{\partial l_{6}}{\partial A_{1 x}} & \frac{\partial l_{6}}{\partial A_{1 y}} & \frac{\partial l_{6}}{\partial A_{2 x}} & \frac{\partial l_{6}}{\partial A_{2 y}} & \frac{\partial l_{6}}{\partial A_{3 x}} & \frac{\partial l_{6}}{\partial A_{3 y}}
\end{array}\right]_{6 \times 6}
$$

where $A_{i x}, A_{i y}(i=1,2,3)$ are the $x$ and $y$ coordinates of three end-effector points in fixed reference frame.

And the dexterity index as

$$
\kappa(\tilde{\boldsymbol{J}})=\|\tilde{\boldsymbol{J}}\|_{2} \cdot\left\|\tilde{\boldsymbol{J}}^{-1}\right\|_{2}=\sqrt{\lambda_{\max }\left(\tilde{\boldsymbol{J}}^{T} \tilde{\boldsymbol{J}}\right) / \lambda_{\min }\left(\tilde{\boldsymbol{J}}^{T} \tilde{\boldsymbol{J}}\right)}
$$

where $\lambda_{\max }(\cdot)$ and $\lambda_{\text {min }}(\cdot)$ represent the maximum and minimum eigenvalues of matrix. It's obviously that $1 \leq \kappa(\tilde{\boldsymbol{J}})<\infty$. When $\kappa(\tilde{\boldsymbol{J}})$ equals 1 , the manipulator is of optimal motion transfer capability and called an isotropy manipulator in this configuration. When $\kappa(\tilde{\boldsymbol{J}})$ becomes huge, the manipulator may approach a singularity configuration. $\kappa(\tilde{\boldsymbol{J}})$ is configuration dependent and in order to evaluate the general performance in the workspace, we use the global condition number 


$$
f_{1}=\frac{\int_{W} \kappa(\tilde{\boldsymbol{J}}) d W}{\int_{W} d W}
$$

which is the condition number integrated over the desired workspace and divided by the volume of the workspace.

\section{DYNAMIC STABILITY AND IsOTROPY INDICES}

The complete dynamic equations of 6-UPS Stewart platform in general form in Cartesian space

$$
\boldsymbol{M}(\boldsymbol{q}) \ddot{\boldsymbol{q}}+\boldsymbol{C}(\boldsymbol{q}, \dot{\boldsymbol{q}}) \dot{\boldsymbol{q}}+\boldsymbol{G}(\boldsymbol{q})=J_{p}^{T} \boldsymbol{F}
$$

The Cartesian stiffness matrix of Stewart mechanism

$$
\boldsymbol{K}_{C}=\boldsymbol{J}_{p}^{T} \boldsymbol{K}_{p}
$$

where $\boldsymbol{K}=\operatorname{diag}\left(\left[k_{1}, k_{2}, \cdots k_{6}\right]\right), \quad k_{i}$ is the equivalent axial linear stiffness of each branch.

Free vibration of the platform around any equilibrium configuration is governed by the following linear perturbation equation

$$
\boldsymbol{M}_{1} \delta \ddot{\boldsymbol{x}}+\boldsymbol{K}_{c} \delta \boldsymbol{x}=0
$$

where

$$
\begin{gathered}
\boldsymbol{M}_{1}=\boldsymbol{M}(\boldsymbol{q}) \cdot\left[\begin{array}{cc}
\boldsymbol{E} & 0_{3 \times 3} \\
0_{3 \times 3} & \boldsymbol{H}
\end{array}\right] \\
\boldsymbol{H}=\left[\begin{array}{ccc}
\cos (\psi) \cos (\theta) & -\sin (\psi) & 0 \\
\sin (\psi) \cos (\theta) & \cos (\psi) & 0 \\
-\sin (\theta) & 0 & 1
\end{array}\right]
\end{gathered}
$$

considering the relationship between the platform angular acceleration and the first and second derivatives of Euler angles.

Assume $\delta \boldsymbol{x}=\boldsymbol{X} e^{i \omega t}$ to be a solution of (8), we get the generalized eigenvalue problem as

$$
\left(\boldsymbol{K}_{C}-\omega^{2} \boldsymbol{M}_{1}\right) \boldsymbol{X}=0
$$

The system has non-trivial solution if coefficient matrix vanishes and the eigenvalue equation is obtained

$$
\operatorname{det}\left(\boldsymbol{K}_{C}-\omega^{2} \boldsymbol{M}_{1}\right)=0
$$

Solving (9)and (10), we get six natural frequencies and corresponding modal vectors for the Stewart platform in a particular configuration. Both kinematic and dynamic effects are included in natural frequencies. The manipulator dynamic stability will gradually decrease if the lowest natural frequency falls, and finally become highly unstable when the lowest natural frequency get close to zero and, therefore, can not restore the configuration if it is perturbed by any amount. Thus, the lowest natural frequency, calculated from (10) can be taken as index for dynamic stability [13].

The dynamic stability index can be defined as the lowest natural frequency in the total workspace

$$
f_{2}=\min _{\boldsymbol{X} \in W} \omega(\boldsymbol{X}, \boldsymbol{\alpha})
$$

Also, the local dynamic isotropy index of Stewart platforms can be defined from natural frequencies [9]

$$
f_{3}=\frac{\omega_{\max }(\boldsymbol{X}, \boldsymbol{\alpha})}{\omega_{\min }(\boldsymbol{X}, \boldsymbol{\alpha})}
$$

We call a parallel manipulator completely isotropic if $f_{3}$ is isotropic i.e. $f_{3}=1$. We can use $f_{3}$ as a local dynamic isotropy measure for an optimal design. In practice, typical configurations such as home configuration and limit configurations are selected to generally take the local dynamic isotropy index into account.

\section{Multi-ObJective Optimizing Model}

The optimizing model base on natural frequencies and dexterity can be obtained

$$
\left\{\begin{array}{l}
\min f_{1}(\boldsymbol{X}, \boldsymbol{\alpha}), f_{2}(\boldsymbol{X}, \boldsymbol{\alpha}), f_{3}(\boldsymbol{X}, \boldsymbol{\alpha}) \\
\text { s.t. } \boldsymbol{\alpha}_{\mathrm{L}} \leq \boldsymbol{\alpha} \leq \boldsymbol{\alpha}_{U} \\
L_{i}^{\min } \leq L_{i}(\boldsymbol{X}, \boldsymbol{\alpha}) \leq L_{i}^{\max } \\
\varphi_{k}^{\min } \leq \varphi_{k}(\boldsymbol{X}, \alpha) \leq \varphi_{k}^{\max } \\
\text { distance }\left(\boldsymbol{L}_{i}, \boldsymbol{L}_{j}\right) \geq R_{i}+R_{j}
\end{array}\right.
$$

Formula (13) is clearly a constrained, nonlinear MOP. Three indices in (5), (11) and (12) as well as the constraints have no explicitly analytical expressions with respect to the set of design variables. Traditional gradient-based methods may not be appropriate in this problem. Weighting method can translate MOPs to single-objective optimization problems by adding weighting factors before different objective functions and popular mathematical programming can manage. However, since the nonlinear, nondifferentiable and discontinuous of objective functions and constraints in our problem, traditional mathematical programming usually works at a low efficiency and always sensitive to weighting factors.

Pareto-dominance based EMO algorithms are usually useful in searching global solutions in MOPs by maintaining potential solutions between different generations. In this paper, two EMO algorithms including the classical NSGA-II [17] and MOPSO-CD [18] were adopted here to search for the Paretooptimal set and frontier.

\section{PeRformance Metrics And Simulation Results}

The two performance metrics used for evaluating the optimization result are the spacing metric[18] and two set coverage (C) proposed by Zitzler[19].

The lower and upper boundaries of design variables in simulation are listed in Tab. I and the following computational parameters are adopted:

Population size: 100

Maximum generations: 2000 
Pareto-optimal set size: 100

Tour size (in NSGA-II): 2

TABLE I. DESIGN VARIABLES BOUNDARIES

\begin{tabular}{|c|c|}
\hline $\begin{array}{c}\text { Design } \\
\text { variables }\end{array}$ & Boundaries \\
\hline$R_{p}$ & $(0.05,0.1) \mathrm{m}$ \\
\hline$R_{B}$ & $(0.12,0.22) \mathrm{m}$ \\
\hline$\lambda_{p}$ & $\left(18^{\circ}, 28^{\circ}\right)$ \\
\hline$\lambda_{B}$ & $\left(12^{\circ}, 22^{\circ}\right)$ \\
\hline$z_{0}$ & $(0.16,0.26) \mathrm{m}$ \\
\hline
\end{tabular}

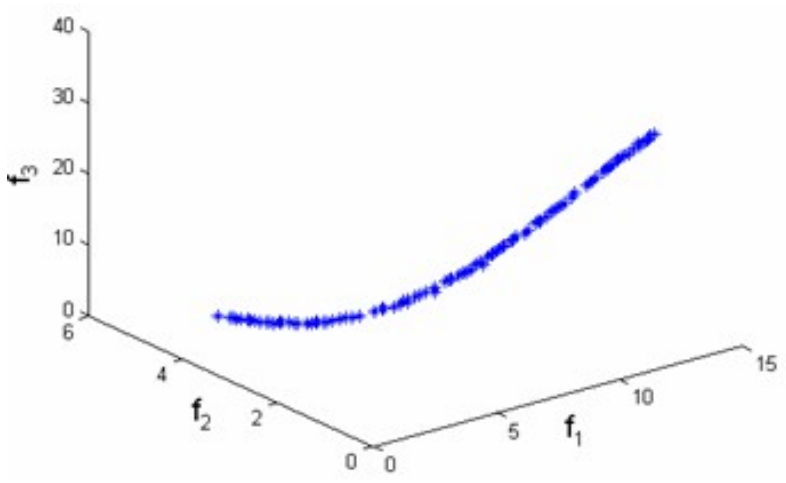

Figure.3 Pareto-optimal frontier generated by NSGA-II

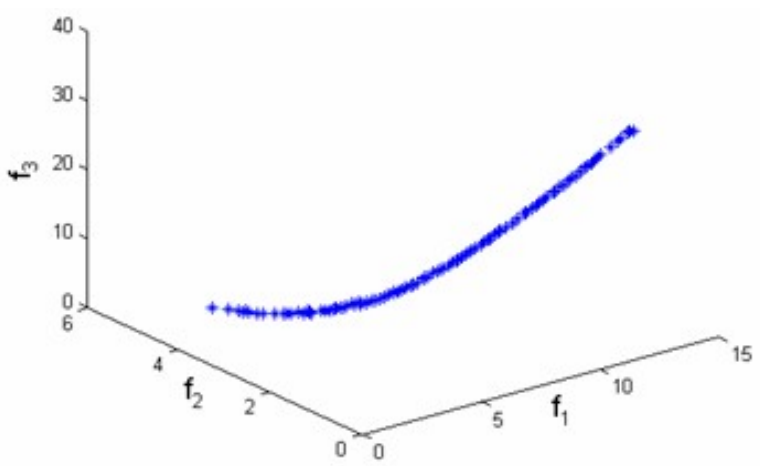

Figure.4 Pareto-optimal frontier generated by MOPSO-CD

Fig. 3 and 4 show the Pareto-optimal frontier after 2000 generations. We can see that both NSGA-II and MOPSO-CD acquired an approximately degenerated hypersurface in the Cartesian coordinates where the coordinates of three axes represent values of the three indices $f_{1}, f_{2}$ and $f_{3}$. Designers can make decisions only by sampling the corresponding Paretooptimal set according to their experiences or preferences. Fig. 5 and 6 show the vertical view of the Pareto-frontier where the lower-left area is beyond the frontier and inaccessible in the solution space, while sampling in the upper-right area of the frontier can only obtain an inferior solution where either condition number or minimum frequency index is larger than the Pareto-optimal solutions.

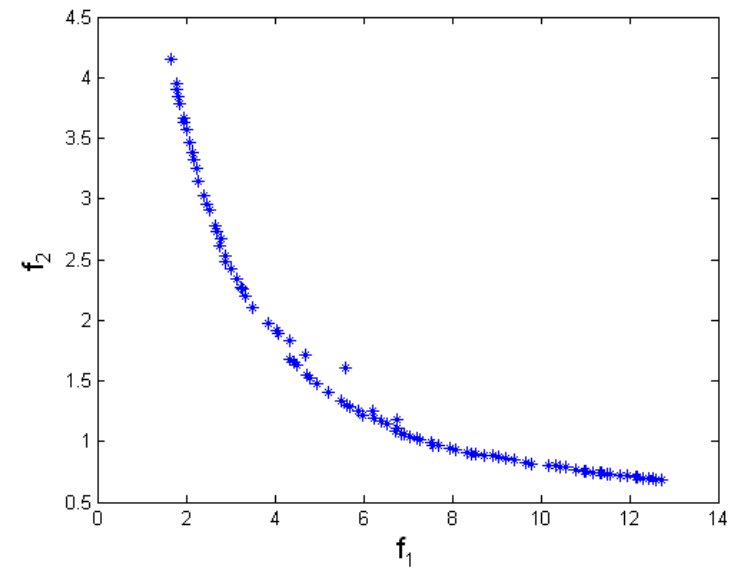

Figure.5 Pareto-optimal frontier generated by NSGA-II (from vertical view)

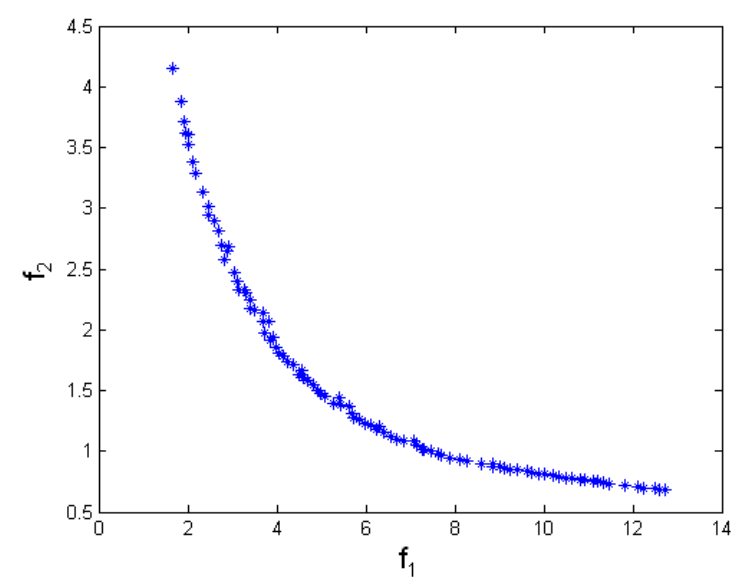

Figure.6 Pareto-optimal frontier generated by MOPSO-CD (from vertical view)

Tab. II shows the results of performance metrics of NSGAII and MOPSO-CD. With regard to the two set coverage metric $(C)$, NSGA-II and MOPSO-CD are incomparable according to the definition. However, in terms of solution diversity, MOPSO-CD has a better distribution of the generated Pareto- optimal solutions than NSGA-II, which can also be seen from figs. 5 and 6 intuitively. Moreover, the computational time of MOPSO-CD is reduced and this may be attributed to the nondominated sorting mechanism in NSGA-II where both ranking positions and crowding distances are computed in the archive, but in MOPSO-CD, only crowding distances are computed.

TABLE II. REsUlts of PERFormance Metrics

\begin{tabular}{|c|c|c|c|}
\hline \multirow{2}{*}{$\begin{array}{c}\text { EMO } \\
\text { Algorithms }\end{array}$} & Coverage metric & $\begin{array}{c}\text { Spacing } \\
\text { metric }\end{array}$ & $\begin{array}{c}\text { Computational } \\
\text { time (in } \\
\text { seconds) }\end{array}$ \\
\cline { 2 - 4 } & $C(\boldsymbol{B}, \boldsymbol{A})=0.06^{\mathrm{a}}$ & 0.212 & 74209 \\
\hline NSGA-II & $C(\boldsymbol{A}, \boldsymbol{B})=0.15$ & 0.161 & 69866 \\
\hline MOPSO-CD & a. $\boldsymbol{A}$ represents the solutions of NSGA-II and $\boldsymbol{B}$ the solutions of MOPSO-CD
\end{tabular}




\section{CONCLUSION}

In this paper, EMO based optimizations have been investigated in order to increase the dynamic performance of Stewart platforms. Three indices, including the dimensionally homogeneous Jacobian matrix condition number, minimum natural frequency in the workspace and local dynamic isotropy index at typical configurations, are introduced as objective functions in multi-objective optimal design.

Two classical Pareto-dominance based EMO algorithms including NSGA-II and MOPSO-CD are adopted to search for the Pareto-optimal frontier of our MOP. Performances of both algorithms are compared by defined coverage and spacing metrics. Results show that NSGA-II and MOPSO-CD obtain two incomparable solution sets. However, MOPSO-CD gets a better spacing performance and, moreover, saves computational time by at least an hour in 2000 computational generations. By synthetically considering, MOPSO-CD algorithm may be more competitive in solving our problem and the results are valuable in designing a Stewart platform for well dynamic performance.

\section{REFERENCES}

[1] Y. J. Lou, G. F. Liu and Z. X. Li, "Optimal Design of Parallel Manipulators via LMI Approach,” Proceedings of IEEE International Conference on Robotics and Automation (ICRA' 03), IEEE Press, Sept. 2003, pp. 1869-1874, doi: 10.1109/ROBOT.2003.1241867.

[2] J. P. Merlet and D. Daney, "Appropriate Design of Parallel Manipulators," Smart Devices and Machines for Advanced Manufacturing, Springer, London, 2008, pp. 1-25, doi: 10.1007/978-184800-147-3_1.

[3] S. Bhattacharya, H. Hatwal and A. Ghosh, "On the Optimum Design of Stewart Platform Type Parallel Manipulators,” Robotica, vol. 13, Mar. 1995, pp. 133-140, doi: 10.1017/S026357470001763X.

[4] F. Gao, F. Guy and W. A. Gruver, "Criteria Based Analysis and Design of Three-degree-of-freedom Planar Robotic Manipulators,” Proceedings of IEEE International Conference on Robotics and Automation (ICRA'97), IEEE Press, Apr. 1997, pp. 468-473, doi: 10.1109/ROBOT.1997.620081.

[5] K. Miller, "Optimal Design and Modeling of Spatial Parallel Manipulators,” The International Journal of Robotics Research, vol. 23, Feb. 2004, pp. 127-140, doi: 10.1177/0278364904041322.

[6] X. J. Liu, “Optimal Kinematic Design of a Three Translational DoFs Parallel Manipulator,” Robotica, vol. 24, Apr. 2006, pp. 239-250, doi:10.1017/S0263574705002079.
[7] C. Gosselin, "Kinematic Analysis Optimization and Programming of Parallel Robotic Manipulators,” PhD Thesis, McGill University, Montreal, Canada, 1988.

[8] G. Nawratil, "New performance indices for 6-dof UPS and 3-dof RPR parallel manipulators,” Mechanism and Machine Theory, vol. 44, Jan. 2009, pp. 208-221, doi: 10.1016/j.mechmachtheory.2008.02.004.

[9] H. Z. Jiang, H. J. Feng and Z. Z. Tong, “Characteristics analysis of joint space inverse mass matrix for the optimal design of a 6-DOF parallel manipulator," Mechanism and Machine Theory, vol. 45, May. 2010, pp.722-739, doi: 10.1016/j.mechmachtheory.2009.12.003.

[10] W. Wang, H. Y. Yang and J. Zou, “Optimal design of Stewart platforms based on expanding the control bandwidth while considering the hydraulic system design,” Journal of Zhejiang University Science A, vol. 10, Jan. 2009, pp. 1862-1775, doi: 10.1631/jzus.A0820329.

[11] F. Hao and J. P. Merlet, "Multi-criteria Optimal Design of Parallel Manipulators Based on Interval Analysis," Mechanism and Machine Theory, vol. 40, Feb. 2005, pp. 157-171, doi: 10.1016/j.mechmachtheory.2004.07.002.

[12] Z. Gao, D. Zhang and Y. J. Ge, "Design Optimization of a Spatial Six Degree-of-freedom Parallel Manipulator Based on Artificial Intelligence Approaches,” Robotics and Computer-Integrated Manufacturing, vol. 26, Apr. 2010, pp. 180-189, doi: 10.1016/j.rcim.2009.07.002.

[13] P. Mukherjee, B. Dasgupta and A. K. Mallik, "Dynamic Stability Index and Vibration Analysis of a Flexible Stewart Platform," Journal of sound and vibration, vol. 307, Nov. 2007, pp. 495-512, doi: 10.1016/j.jsv.2007.05.036.

[14] J. K. Slisbury and J. J. Craig, "Articulated hands: force control and kinematic issues,” International Journal of Robotics Research, vol. 1, Mar. 1982, pp. 4-17, doi: 10.1177/027836498200100102.

[15] G. Pond and J. A. Carretero, "Formulating Jacobian matrices for the dexterity analysis of parallel manipulators," Mechanism and Machine Theory, vol. 41, Dec. 2006, pp. 1505-1519, doi: 10.1016/j.mechmachtheory.2006.01.003.

[16] S. G. Kim and J. Ryu, “New Dimensionally Homogeneous Jacobian Matrix Formulation by Three End-effector Points for Optimal Design of Parallel Manipulators,” IEEE Transactions on Robotics and Automation, vol. 19, Aug. 2003, pp. 731-736, doi: 10.1109/TRA.2003.814496.

[17] K. Deb, A. Pratap and S. Agarwal, "A Fast Elitist Multiobjective Genetic Algorithm: NSGA-II,” IEEE Transactions on Evolutionary Computation, vol. 6, Apr. 2002, pp.182-197, doi: 10.1109/4235.996017.

[18] C. R. Raquel and P. C. Naval Jr, "An Effective Use of Crowding Distance in Multiobjective Particle Swarm Optimization,” Proceeding of Genetic and Evolutionary Computation Conference (GECCO’05), ACM press, June, 2005, pp. 257-264, doi: 10.1145/1068009.1068047.

[19] E. Zitzler, M. Laumanns and L. Thiele, "SPEA2: Improving the strength pareto evolutionary algorithm,” TIK-Report 103, May. 2001, Swiss Federal Institute of Technology. 